\title{
Diagnostic nerve biopsy: a case history
}

\author{
John W Griffin
}

Diagnostic nerve biopsy provides an instructive case history of the developmental arc of a laboratory test. Biopsy of peripheral nerves became a clinically applicable procedure around 40 years ago, and the techniques were developed in the laboratories of pioneers such as Peter Dyck, PK Thomas and Arthur Asbury. In the 20 years that followed, nerve biopsies taught us much about the histopathological basis of nerve disease, and the latest findings were presented at well-attended sessions at annual neurology meetings. We learned the pathology of the inflammatory demyelinating neuropathies, the nature of demyelination and onion-bulb formation in Charcot-Marie-Tooth disease type 1 (CMT1), the features of various types of vasculitic neuropathy, the range of pathology in diabetic nerve injury, and the pathology of various less-prevalent disorders.

The techniques required to fully evaluate a specimen-plastic embedding of large specimens, preparation of teased nerve fibers, immunocytochemistry, morphometry, and paraffin histology - are complex, and are not the province of routine pathology laboratories. The few laboratories that chose to focus specifically on nerve biopsy studied large numbers of samples and developed the technical aspects to a high art.

Over the past 20 years, new findings have become fewer, and the clinical indications for nerve biopsy have been narrowed by the development of other newer, less-invasive technologies. High-quality electrophysiology has become more widely available, and can give clues to inflammatory or heritable etiologies, as well as suggesting specific diagnoses such as neuropathy associated with antibodies against myelin-associated glycoprotein. Genetic testing has largely eliminated the need for nerve biopsy in the diagnosis of heritable neuropathies such as CMT1 and some types of amyloidosis. Serological tests can recognize many paraneoplastic neuropathies, neuropathies associated with monoclonal gammopathies, and other disorders. Skin biopsies have provided a new window into the

\section{A technically \\ inadequate \\ biopsy is a \\ substantial \\ disservice to \\ our patients}

JW Griffin is the Editor-in-Chief of Nature Clinical Practice Neurology.

Competing interests

The author declared no competing interests.

www.nature.com/clinicalpractice doi:10.1038/ncpneuro0776 involvement of small sensory and autonomic fibers in neuropathies. Nerve biopsies were never ideal for this purpose, because they required cumbersome electron micrographic assessment, and they provided only one snapshot in space and time. By contrast, skin biopsies can be performed at multiple sites and repeated as needed. There are still some major clinical indications for nerve biopsy, however, including diagnosis of vasculitis of nerve, some cases of amyloid neuropathy (after screens for light-chain proteinuria and prevalent mutations of familial amyloidosis), and multifocal leprosy. Nerve biopsy also remains a 'last resort' for identification of unexpected or rare neuropathies, such as giant axonal neuropathy, in difficult diagnostic cases.

Despite the narrowing of the indications for nerve biopsy, the number of laboratories willing to undertake processing of nerve biopsies has risen, at least in the US. Perhaps as a consequence, the quality of processing has become more variable. A technically inadequate biopsy is a substantial disservice to our patients. Biopsy of a nerve such as the sural nerve is an invasive procedure and should be a 'one-per-customer' undertaking; only in extraordinary situations should it be necessary or appropriate to recommend a second biopsy. As neurologists we have two roles. First, we need to make sure that biopsy is appropriate for the patient and that we are clear about what we are seeking. Second, we need to make sure that both the surgeon and the processing laboratory are experienced. How many nerve biopsies do they process annually? Does the laboratory do plastic sections, fiber teasing, and other specialized approaches? In countries in which special certification in nerve or neuromuscular pathology is available, is the laboratory certified, or does it have arrangements with other specialized laboratories to handle the processing?

Nerve biopsy remains a valuable technique in selected patients, but we need to ensure that the right patients undergo biopsy, and that the maximum amount of information is obtained from the procedure. 